\title{
Da (inter)corporeidade à depressão melancólica na psicopatologia fenomenológica de Fuchs
}

\author{
From (inter)corporeality to melancholic depression on Fuchs' \\ phenomenological psychopathology
}

\author{
Victor Monteiro \\ Camila Souza \\ Virgínia Moreira \\ Universidade de Fortaleza \\ Brasil
}

\begin{abstract}
Resumo
Neste artigo, tivemos como objetivo revisar os fundamentos teóricos da psicopatologia fenomenológica de Thomas Fuchs no que tange a experiência do corpo na depressão melancólica. Através de pesquisas em bases de dados eletrônicas, realizamos uma revisão de literatura que investigou as obras publicadas pelo autor entre 1994 e 2018. Encontramos a corporeidade destacada como uma via de acesso à compreensão da depressão melancólica, desenvolvida no sentido da intercorporeidade ao se centrar na ideia de intersubjetividade. A intercorporeidade é a ponte de constituição ambígua da relação homem-mundo, possibilitando o compartilhamento de nossas experiências. Para Fuchs, a depressão melancólica é um transtorno da intercorporeidade, pois ao invés de conectar homem e mundo a experiência corporal torna-se um obstáculo. Fuchs contribui para a construção de uma perspectiva de humano encarnado, em que este se configura como unidade indivisível na experiência ao compreender a depressão melancólica como modo de estar no mundo do paciente.

Palavras-chave: psicopatologia fenomenológica; corporeidade; intercorporeidade; depressão melancólica; Thomas Fuchs.
\end{abstract}

\begin{abstract}
In this paper, we aimed to review the theorical foundations of Thomas Fuchs' phenomenological psychopathology regarding the experience of the body in melancholic depression. Through research in electronic databases, we performed a literature review which investigated papers published by the author between the years of 1994 and 2018. We've found the corporeality highlighted as an access way to understanding melancholic depression, developed towards intercorporeality by focusing on the idea of intersubjectivity. Intercorporeality is the bridge of ambiguous constitution of the human-world relation, allowing the sharing of our experiences. For Fuchs, melancholic depression is a disorder of intercorporeality, because instead of connecting human and world, the bodily experience becomes an obstacle. Fuchs contributes for the construction of an embodied human perspective; in which it is set as an indivisible unit in the experience by comprehending melancholic depression as a patient's way of being in the world.
\end{abstract}

Keywords: Phenomenological Psychopathology; corporeality; intercorporeality; melancholic depression; Thomas Fuchs. 
Com origens no contexto psiquiátrico europeu do século $\mathrm{XX}$, a psicopatologia fenomenológica surge inspirada na fenomenologia filosófica e nas filosofias existenciais. Ela visa à compreensão da experiência vivida do paciente e a investigação das estruturas basilares de sua existência, tais como a relação com o corpo, com o espaço, com o tempo e com o Outro ${ }^{1}$ (Bloc, Souza, \& Moreira, 2016; Fuchs, 2010a; Minkowski, 1927/1997; Tatossian, 1979/2006). É uma proposta de compreensão do adoecimento mental que não se restringe ao modelo sintomatológico e estatístico predominante na psiquiatria neopositivista, apresentando valiosas contribuições aos estudos dos transtornos mentais na contemporaneidade.

Nas últimas décadas, com o crescente número de casos de depressão (World Health Organization, 2018), clínicos e pesquisadores da psicopatologia fenomenológica vêm priorizando estudos voltados à compreensão do vivido depressivo. Dentre eles, destaca-se o psiquiatra alemão Dr. Thomas Fuchs (PhD), que ocupa, atualmente, o cargo de professor na Universidade de Heidelberg, na Alemanha. O trabalho científico de Fuchs tem como prioridade promover o estudo das patologias sob o referencial da fenomenologia filosófica e da tradição em psicopatologia fenomenológica, tendo entre suas influências a filosofia de Maurice Merleau-Ponty.

Fuchs nasceu em 29 de setembro de 1958 na cidade de Munique, na Alemanha. Concluiu seus estudos nos campos da medicina, da filosofia e da história da ciência em 1988, formando-se como especialista em psiquiatria em 1996 pela Universidade Técnica de Munique. Em 2005, tornou-se professor da Universidade de Heidelberg e passou a se dedicar ao ensino dos fundamentos filosóficos da psiquiatria e da psicoterapia, assumindo o cargo do célebre professor, filósofo e psiquiatra Karl Jaspers.

Atualmente, a produção científica de Fuchs corresponde a mais de 200 publicações, entre artigos científicos, livros e capítulos de livro nos idiomas alemão e inglês. Dada a extensa obra do autor, realizamos uma pesquisa de revisão narrativa dos escritos de Thomas Fuchs, tendo como critérios inclusão os trabalhos escritos em inglês, com texto completo disponível para download e que apresentassem no título, no resumo ou nas palavras-chave o termo "depression". Nas plataformas Periódicos CAPES, SciELO, Ebsco host, Scopus, Web of Science e ScienceDirect localizamos um total de 26 fontes que, removendo suas duplicatas, resultaram em 16 artigos da autoria de Fuchs.

1 O termo "Outro" é utilizado em maiúsculo no campo da psicopatologia fenomenológica, como alusão à relação intersubjetiva sujeito/mundo, e optamos por manter a grafia original. 
Na plataforma de pesquisa ResearchGate, entretanto, identificamos mais um artigo de acordo com os critérios estabelecidos na pesquisa e que não estava compilado nas bases de dados investigadas. Com isto, obtivemos 17 artigos publicados no período de 1994 e 2018. Em razão da amplitude da obra do autor e da complexidade de seu pensamento, também investigamos outras fontes que não apresentaram o termo "depression" no resumo, no título ou nas palavras-chave, mas que de algum modo expressam o modo o autor de pensar a psicopatologia e a intercorporeidade. Acrescidas estas fontes, investigamos o total de 45 publicações, incluindo um livro recentemente traduzido para o português (Fuchs, 2018b).

Nos textos estudados encontramos o tema da corporeidade ocupando um lugar de destaque nas discussões de Fuchs no campo da psicopatologia fenomenológica. No caso da depressão melancólica, Fuchs (2001a; 2002b; 2002c; 2005a; 2005c; 2005d; 2005e; 2007b; 2010a; 2010c; 2012a; 2012b; 2013a; 2013b; 2013c; 2014a; 2014b, Fuchs \& Schlimme 2009) descreve uma perturbação no movimento pré-reflexivo e fluido do corpo vivido, o que culmina na alienação e despersonalização do paciente em sua relação consigo mesmo, com os outros e com o mundo ao nível da interafetividade.

Neste artigo ${ }^{2}$, tivemos objetivo revisar os fundamentos teóricos da psicopatologia fenomenológica de Thomas Fuchs no que tange a experiência do corpo na depressão melancólica. Para isto, apresentamos inicialmente a compreensão do autor sobre a dimensão da (inter)corporeidade e seu papel na constituição da relação homem-mundo. Em seguida, descrevemos a experiência do corpo na depressão melancólica sob a lente de Fuchs, que a caracteriza como um transtorno da dimensão afetiva da intercorporeidade.

\section{A corporeidade}

Thomas Fuchs reconhece a dimensão pré-reflexiva do corpo como mediadora da experiência, o que nos possibilita a conexão com os outros e com o mundo. Parte da distinção fenomenológica entre dois modos de experienciar o corpo. São eles: o corpo vivido ou corpo-sujeito (Lieb) e o corpo-objeto (Körper), que se apresentam como elementos fundamentais da constituição ambígua da

\footnotetext{
2 Este trabalho foi desenvolvido a partir de financiamentos do Conselho Nacional de Desenvolvimento Científico e Tecnológico (CNPq) e da Coordenação de Aperfeiçoamento de Pessoal de Nível Superior (CAPES), por meio de, respectivamente, uma bolsa de iniciação cientifica (PIBIC) e uma bolsa de doutorado. Este trabalho também é vinculado ao projeto PQ-1D CNPq "Fenomenologia Clínica da Intersubjetividade no mundo vivido (Lebenswelt) psicopatológico" (Financiamento Edital 05/2016 Auxílio Complementar à Pesquisa na Universidade de Fortaleza - UNIFOR, projeto 1965).
} 
corporeidade (Fuchs, 2001b; 2002a; 2002c; 2005a; 2005e; 2010a; 2013a; 2014b; 2015b; Fuchs \& Schlimme, 2009; Fuchs \& De Jaegher, 2009; Fuchs \& Koch, 2014).

Fuchs não concebe estes modos em uma relação dualista ou dicotômica, pois compreende a experiência do corpo como oscilação entre o corpo-sujeito e corpoobjeto, isto é, entre um modo implícito e outro explícito da corporeidade. Algumas patologias mentais, portanto, apresentariam algum tipo de perturbação na vivência destas dimensões (Fuchs, 2010a), o que leva o autor a se dedicar à compreensão da experiência destes modos corporais em contextos patológicos diversos (de Haan \& Fuchs, 2010; Gaete \& Fuchs, 2016; Fuchs, 2001a; 2001b; 2002a; 2002c; 2005a; 2005b; 2005c; 2005d; 2005e; 2006; 2007c; 2010a; 2010b; 2012b; 2013a; 2013b; 2013c; 2014a; 2014b; 2015a; 2015b; 2015c; 2018a; 2018b; Fuchs \& Duppen, 2017; Fuchs \& Koch, 2014; Fuchs \& Schlimme, 2009; Hirjak, Breyer, Thomann \& Fuchs, 2013), tais como a depressão melancólica.

\section{O corpo vivido como mediador tácito: o fundamento da intercorporeidade}

Nas filosofias de Husserl (1954/2014) e Merleau-Ponty (1942/2006; 1945/2011; 1964/2014), embora vejamos uma compreensão do corpo-sujeito (Lieb) e corpo-objeto (Körper) enquanto componentes do corpo vivido, na obra de Fuchs observamos que estes conceitos são apresentados de modo distinto, de tal forma que ao tratar de corpo vivido o psiquiatra alemão esteja se referindo apenas ao corpo-sujeito. No entendimento de Fuchs (2002c; 2005e; 2010a; 2013a; 2014b; Fuchs \& Schlimme, 2009), o corpo vivido remete apenas ao modo pré-reflexivo da corporeidade, em que nos movemos e interagimos com os outros sem determos nossa atenção sobre como efetivamos o mover e o interagir. Seria o corpo tácito manifesto em nossas performances habituais, efetuadas sem a necessidade de um esforço reflexivo sobre o próprio corpo, desenrolando-se de modo espontâneo e imediato.

Se for preciso que eu me locomova de um local a outro, por exemplo, e esta locomoção já me for habitual, não reflito nos detalhes dos movimentos de meus pés e tampouco sobre a curvatura na qual ajusto minha coluna. Ao contrário, apenas me dirijo aos fins que almejo; sem pensar nos elementos que compõem meus movimentos. Neste processo, o corpo não é para mim objeto de reflexão, mas um ponto-zero de direcionamento que é em si mesmo despercebido explicitamente. Carrego comigo apenas uma consciência implícita de sua presença, dirigindo-me no mundo livremente (Fuchs, 2002c; 2005e; 2010a; 2013a; 2014b; Fuchs \& Schlimme, 2009). 
$\mathrm{Na}$ medida em que são praticados e repetidos cotidianamente, os movimentos do corpo se tornam habituais e são efetuados com uma espontaneidade e fluidez que não nos demandam a atenção em nós mesmos. Como coloca Fuchs (2005a), "nós não temos que pensar em como fazemos algo, e somos livres para nos direcionar aos fins que escolhemos" (p. 97 - tradução nossa). Deste modo, o corpo vivido carrega um conhecimento tácito da experiência; um saber implícito reconhecido na relação homem-mundo. Ele é construído à medida que certos movimentos se tornam partes de nossas disposições e repertórios corporais, sendo executados por nós em um só golpe (Fuchs, 2001b; 2005a).

O mundo se desvela a nós corporalmente, de tal modo que nos enraizamos ao mundo e nos organizamos nele a partir do corpo. Para Fuchs (2002c; 2005a; 2013a), o corpo vivido é o mediador da experiência e o responsável por estabelecer a relação existente entre mim e meu entorno. Sua mediação é marcada pela peculiaridade de uma participação pré-reflexiva, que nos revela o mundo sem ser notada explicitamente. Sua presença é sentida de modo tácito a fim de não interromper o acesso ao mundo, apresentando-se enquanto um mediador transparente da experiência (Fuchs, 2005a; de Haan e Fuchs, 2010).

Para ilustrar isto, Fuchs (2005a) traz o exemplo do ato de colocar as mãos sobre o casco de uma árvore. Ao tocá-lo, não só tenho a percepção explícita de sua textura, mas também possuo a consciência implícita de que é a minha mão que o toca. Nesta experiência, não tomo consciência de minha mão (corpo) para posteriormente me dar conta do casco (mundo) - o que tornaria a experiência mecânica - mas percebo a textura do casco diretamente. Caso a percepção de minha mão fosse tornada alvo de minha atenção, distrair-me-ia para perceber o que toco, alienando-me de presença do mundo. Para que isto não ocorra, o corpo vivido assume uma participação pré-reflexiva, revelando o mundo na medida em que 'oculta' a si próprio. É partindo desta compreensão, que o psiquiatra caracteriza o corpo vivido como um mediador transparente, como metáfora a própria manifestação tácita do corpo no estabelecimento do contato com o mundo (Fuchs, 2005a).

Na construção desta perspectiva, Fuchs faz referência à fenomenologia de Merleau-Ponty, (Fuchs, 2001b; 2002a; 2002c; 2005a; 2005b; 2010a; 2013a; Fuchs \& Schlimme, 2009; de Haan \& Fuchs, 2010; Fuchs \& Koch, 2014) explicitando a influência deste filósofo em suas ideias. Merleau-Ponty (1945/2011) reconhece o corpo como o "veículo do ser no mundo" (p. 122) e meio de comunicação com este, entendendo que, enquanto corpo tocante, este só é visível a mim intuitivamente, permanecendo apenas como horizonte de minha 
experiência (Merleau-Ponty, 1942/2006; 1945/2011; 1964/2014). Ao tratar do corpo, diz Merleau-Ponty:

O que o impede de ser alguma vez objeto, de estar alguma vez "completamente constituído", é o fato de ele ser aquilo por que existem objetos. Ele não é nem tangível nem visível na medida em que é aquilo que vê e aquilo que toca (Merleau-Ponty, 1945/2011, p. 136).

Na psicopatologia fenomenológica de Fuchs (2013a), esta mediação tácita do corpo vivido também se mostra como base à compreensão da experiência afetiva. O psiquiatra alemão se opõe a ideia das emoções como fenômenos psíquicos internos (Fuchs \& Koch, 2014), contrapondo-se a perspectiva dualista herdeira do platonismo e do cartesianismo. De outro modo, as considera visíveis na expressão corporal desde os gestos mais rudimentares, manifestando o modo como experienciamos o mundo. As emoções seriam respostas afetivas a certos eventos que implicam em alterações corporais perceptíveis, como tremor, exaustão ou relaxamento, o que nos mobilizam a ação. Elas são sempre intencionais e expressam o modo como percebemos as situações, atribuindo-lhes significados que estas não teriam em si mesmas (Fuchs, 2013a; Fuchs \& Koch, 2014).

Nós sentimos algo 'no ar', ou sentimos um 'clima' interpessoal, por exemplo, uma atmosfera serena, solene ou ameaçadora [...]. Este espaço emocional é essencialmente sentido através da mediação do corpo a qual amplia, comprime, enfraquece, estremece, chacoalha etc. em correspondência às sensações e atmosferas que experienciamos (Fuchs, 2013a, p. 222 - tradução nossa).

Fuchs e Koch (2014) reconhecem as sensações corporais como componentes inerentes da experiência afetiva, que sempre se move e é movida em relação ao mundo e aos outros. Nisto, o corpo vivido, enquanto mediador da experiência, também assume a função de um ressoador afetivo das vibrações do mundo. Ele reverbera as sensações que exprimem o modo pelo qual o sujeito é afetivamente tocado, a saber, a ressonância corporal (Fuchs, 2013a). Como ilustração, Fuchs (2018b) coloca que sentir medo, por exemplo, seria impossível sem a sensação de uma tensão corporal, de um pulsar do coração, de uma falta de fôlego ou de uma tendência ao afastamento, pois é através delas que nos dirigimos em uma situação ameaçadora. Em razão disto, o autor reconhece que as sensações vividas no corpo não são meros subprodutos das emoções, mas a própria mediação corporal de uma intencionalidade afetiva.

Fuchs e De Jaegher (2009) entendem a interação intersubjetiva enquanto um encontro de corpos vividos, em que eu e o outro nos compreendemos mutuamente 
pela percepção de expressões gestuais, faciais e posturais, as quais se executam como performances corporais pré-reflexivas. Nelas há uma relação de coordenação mútua em que ambos os sujeitos afetam e são afetados reciprocamente, configurando, neste caso, uma ressonância intercorporal (Fuchs, 2013a; 2018b; Fuchs e De Jaegher, 2009; Fuchs e Koch, 2014).

Isto se dá de tal forma que Fuchs e De Jaegher (2009) discorrem sobre uma incorporação mútua entre dois sujeitos intencionais, na qual ambos entram em um estado corporal díade. "Seus esquemas corporais e experiências corporais se expandem e, de certo modo, incorporam o corpo percebido do outro" (p. 472 tradução nossa). Ambos os sujeitos compartilham, deste modo, um mesmo espaço intercorporal, coordenando-se e se sincronizando junto ao corpo vivido do outro. Nesse encontro, flutuamos em um estado ambíguo entre o corpo incorporado do outro e nossa própria posição corporal, o que constitui as esferas da intercorporeidade e, mais precisamente, da interafetividade (Fuchs, 2013a; Fuchs \& De Jaegher, 2009).

Ao tratar destas esferas, Fuchs (2001b; 2002a; 2005a; 2010a; 2014b; 2015b; 2018b; Fuchs \& De Jaegher 2009; Fuchs, \& Koch, 2014) apresenta novamente uma influência da filosofia de Merleau-Ponty como referencial à compreensão da intercorporeidade. Para o filósofo, a experiência intersubjetiva se baseia no fenômeno de transferência do esquema corporal ${ }^{3}$, um processo de percepção corporal do outro e do próprio corpo de modo não-inferencial, no qual se estabelece entre ambos uma influência recíproca. Afirmará que, na relação interpessoal, "tudo se passa como se a intenção do outro habitasse meu corpo ou como se minhas intenções habitassem o seu" (Merleau-Ponty, 1945/2011, p. 251) - colocação esta que vem a ser citada por Fuchs em alguns de seus textos (Fuchs, 2005a; Fuchs \& Schlimme, 2009; Fuchs \& Koch, 2014).

Nisto, Merleau-Ponty (1945/2011) entende a relação homem e mundo, e entre eu e o outro, por uma conexão anterior à operação reflexiva da lógica cartesiana, tomando a intercorporeidade como base para a intersubjetividade. Em sua obra póstuma O Visível e o Invisível, Merleau-Ponty (1964/2014) continua a insistir nesta perspectiva de uma sinergia entre os corpos argumentando que, assim como nosso corpo é capaz de efetivar uma unidade pré-reflexiva e préobjetiva dos sentidos de seus órgãos, os corpos dos organismos também

\footnotetext{
3 Na filosofia de Merleau-Ponty (1945/2011), o esquema corporal é concebido como "uma tomada de consciência global de minha postura no mundo intersensorial [...]" (p. 145) e, portanto, uma experiência de meu corpo no mundo. Logo, ao tratar de uma transferência do esquema corporal, o filósofo aponta para um processo antepredicativo que possibilita o compartilhamento de experiências, e, assim, a constituição de uma intercorporeidade.
} 
realizariam uma influência mútua sobre si, constituindo uma mesma experiência intercorporal.

Resgatando esta noção para compreender a experiência afetiva, Fuchs (2013a, 2018b) concebe toda afetividade enquanto intencional e corporificada, definindo-a em termos de uma interafetividade. Pode-se dizer que as emoções não se constituem como epifenômenos dentro do sujeito, mas enquanto experiências entre os sujeitos e para um mundo intersubjetivo. O corpo, enquanto um ressoador afetivo, expressa a afetividade em suas múltiplas performances, sempre se dirigindo ao mundo, sendo tocado por ele e participando dele com os outros (Fuchs \& Koch, 2014).

Ao seguir a concepção de homem como ser engajado no mundo por meio do corpo, a obra de Fuchs mostra sua inspiração na filosofia merleau-pontyana, em que a experiência da corporeidade não se resume a um vivido implícito do corpo, mas também a uma percepção explícita e notória, que emerge em uma experiência de interrupção desta mediação tácita; fazendo surgir, assim, o corpo-objeto.

\section{O corpo-objeto e a corporalização}

Diferentemente do corpo vivido, o corpo-objeto ${ }^{4}$ faz menção a nossa estrutura física propriamente dita, isto é, ao corpo anatômico, utilizado como objeto de estudo pelas ciências médicas e biológicas (Fuchs, 2002c; 2010a; Fuchs $\&$ De Jaegher, 2009). Ele é percebido explicitamente enquanto uma coisa entre outras coisas; um objeto ao qual nos voltamos e que também pode ser observado pelas pessoas. Neste sentido, é frequentemente percebido por nós como um instrumento a ser manuseado ou manipulado, emergindo na experiência quando o movimento fluido e pré-reflexivo do corpo vivido é interrompido (Fuchs \& Schlimme, 2009).

O corpo objeto aparece sempre que uma reação ou resistência surge à performance primária do corpo-vivido; quando o corpo perde sua coerência pré-reflexiva e automática com o mundo ao redor; quando nossas expressões corporais espontâneas são perturbadas, bloqueadas, ou objetificadas por uma inversão de nossa atenção sobre nós mesmos (Fuchs, 2002c, p. 225 - tradução nossa).

\footnotetext{
${ }^{4}$ Em sua obra, Thomas Fuchs utiliza ambos os termos corporeal body e object-body para se referir a noção de corpo-objeto (Körper). Em razão das possíveis confusões com a tradução literal do primeiro termo para o idioma português (corpo corporal), as citações diretas que utilizam corporeal body no original são traduzidas neste artigo como 'corpo-objeto'.
} 
Com isto, surge na experiência uma percepção materializada e reificada do próprio corpo, tornando-o, deste modo, um corpo-objeto. Por este motivo, Fuchs (2002c; 2005a; 2010a; Fuchs \& Schlimme, 2009) considera que a corporeidade se constitui na relação de entrecruzamento de um modo implícito e outro explícito, os quais estabelecem um constante movimento de oscilação. De acordo com o autor, seria um erro conceber o corpo-objeto como um "corpo estrangeiro" (Fuchs, 2002c, p. 224 - tradução nossa) de nossa experiência ou mero resultado de nossa reflexão objetificante, uma vez que, sob este viés, construiríamos um novo dualismo substituto da antiga cisão cartesiana entre mente e corpo (Fuchs, 2002c).

Seguindo por outro caminho, Fuchs (2002c) afirma que o corpo corporal vem a ser experienciado em situações específicas nas quais, em geral, vivencia-se uma situação de desconforto no manuseio do próprio corpo. Quando sentimos que não somos capazes de efetuar determinado movimento, ou quando nos percebemos como alvo de atenção do olhar dos outros. Nestes contextos, permanecemos atentos a nós mesmos, deixando de tomar o corpo como um ponto zero de direcionamento. Fuchs (2002c; 2005a; Fuchs \& Schlimme, 2009) nomeará este fenômeno corporalização (corporealization), uma experiência da realidade voltada ao corpo e à sua estrutura física.

Ao nos sentirmos exaustos, por exemplo, movemos nosso corpo com dificuldade, como um objeto pesado que carregamos de um lado ao outro. 0 percebemos como uma estrutura sólida que precisa ser empurrada; e não mais como um corpo transparente e pré-reflexivo. Nossa atenção e esforço se voltam para como o utilizamos, fazendo-o ser percebido por nós como frágil, obstinado ou preguiçoso. De outro modo, isto pode ser observado quando tentamos aprender um movimento novo, como uma dança desconhecida por nós. Neste caso, colocamo-nos atentos para como estamos utilizando nosso próprio corpo em cada passo realizado, vindo a percebê-lo enquanto uma difícil ferramenta a ser manuseada, e, portanto, como objeto de nossa atenção. Em ambos os casos, nossos corpos se corporalizam em corpo-objeto, uma vez que, em decorrência do cansaço e da não familiaridade com a dança, o corpo-objeto que 'possuo', por um momento, sobrepõe-se ao corpo vivido que 'sou', alterando a experiência de minha corporeidade (Fuchs, 2005a).

Teríamos assim constituída uma oscilação na corporeidade, em que um modo intuitivo e outro modo reflexivo se alteram em nossas experiências cotidianas. Nosso corpo, portanto, seria, como colocado por Merleau-Ponty (1945/2011), um corpo ambíguo, que se mostra enquanto aquele que vê e que sofre simultaneamente. Ele é um ser de duas faces, o qual é ao mesmo tempo coisa 
entre as coisas e também aquilo que as vê e as toca, constituindo-se como sujeito e objeto (Merleau-Ponty, 1964/2014).

Se o que se quer são metáforas, seria melhor dizer que o corpo sentido e o corpo que sente são como o direito e o avesso, ou ainda, como dois segmentos de um único percurso circular que, do alto, vai da esquerda para a direita e, de baixo, da direita para a esquerda, constituindo, todavia, um único movimento em suas duas fases (Merleau-Ponty, 1964/2014, p. 134).

Não se restringindo à análise da experiência pré-reflexiva do corpo, Fuchs apresenta a corporeidade em sua constituição ambígua com o mundo e com o outro, e, como veremos adiante, ela se mostra distorcida na experiência patológica da depressão melancólica.

\section{A depressão melancólica}

Para ilustrar as manifestações da corporeidade nas experiências de adoecimento, encontramos um rico cenário de discussão sobre a depressão melancólica nos estudos fenomenológicos de Fuchs (2001a; 2002b; 2002c; 2005a; 2005c; 2005d; 2005e; 2007b; 2010a; 2010c; 2012a; 2012b; 2013a; 2013b; 2013c; 2014a; 2014b, 2018a, 2018b; Fuchs \& Schlimme 2009; Fuchs \& Duppen, 2017). Na literatura investigada, entretanto, suas discussões fenomenológicas sobre o transtorno só se iniciam a partir do ano de 2001. Antes disso, havia apenas estudos empíricos sobre depressões endógenas e parafrenias, os quais não se propunham a investigar o vivido depressivo sob a lente da fenomenologia (Fuchs, 1994, 1999a, 1999b).

Nos anos de 2004, 2008 e 2011, Fuchs também é coautor de pesquisas empíricas voltadas ao estudo da depressão pós-parto, mas também não desenvolve um olhar fenomenológico sobre este quadro específico (Reck e outros, 2004; Reck e outros, 2008, Reck e outros, 2011). Em 2016, Cutting, Moratidou, Fuchs e Owen (2016) discutem as possíveis influências de Max Scheler sobre a noção de depressão vital em Kurt Schneider, tratando-se apenas de um artigo de revisão teórica que não expressa elaborações próprias de Fuchs.

Nas demais obras, observamos que Fuchs usa de maneira indistinta os termos depressão, melancolia e depressão melancólica para discorrer sobre o vivido depressivo. Entretanto, na tradição da psicopatologia fenomenológica, o termo depressão melancólica faz alusão ao estado psicótico deste quadro clínico (Tatossian, 1979/2006). Ao fazer menção a este estado da depressão, Fuchs (2001a; 2002c; 2005a; 2005c; 2005e; 2010a; 2013a; 2013c; 2014b; Fuchs \& 
Schlimme, 2009) se utiliza dos termos psiquiátricos Síndrome de Cotard ou melancholia anaesthetica, referentes a uma psicopatologia depressiva grave acompanhada da presença de delírios e alucinações.

$\mathrm{Na}$ psicopatologia fenomenológica de Fuchs (2005e; 2010a; 2013a), a depressão melancólica é apresentada como perturbação na experiência do corpo vivido, isto é, um transtorno da intercorporeidade ou, mais precisamente, da interafetividade. Para Fuchs (2002c; 2013a; 2014b), o corpo vivido na depressão perde sua mobilidade e fluidez, sendo sentido de modo sólido e rígido na experiência. Ao invés de conectar o sujeito ao mundo e aos outros, ele se torna um obstáculo para todos os impulsos do indivíduo, configurando um estado patológico de corporalização ou hipercorporificação (Fuchs, 2002c; 2005a; 2005e; 2010a; 2013a; 2014b). O depressivo experiencia um corpo pesado, como um estorvo que dificulta a realização de seus movimentos e de seu direcionamento ao mundo (Fuchs, 2002c; 2005a). Seus gestos e performances corporais, outrora leves e espontâneos, tornam-se lentos e de difícil execução; o que vem a ser expresso nas sensações de fadiga e laxidão do indivíduo (Fuchs, 2002c; 2005e; 2014b).

Com este enrijecimento, a ressonância corporal do depressivo é impossibilitada, não permitindo a ele a realização de intercâmbios afetivos com seu ambiente. Tendo sido corporalizado, seu corpo perde a capacidade de se engajar afetivamente ao mundo, havendo uma experiência de alienação em suas relações sociais. Os gestos e expressões corporais das pessoas já não são mais capazes de o atrair ou afetar, o que o impede de se conectar com os outros (Fuchs, 2010a; 2013a; Fuchs \& Koch, 2014).

O corpo depressivo carece de expressão e não oferece pistas a percepção empática dos outros. A contínua sincronização dos gestos corporais e olhares que normalmente acompanham a interação colapsam. [...]. Logo, eles [os pacientes], sentem-se incapazes de comunicar sua experiência e tentam em vão compensar a perda de ressonância através da repetição estereotipada de seus descontentamentos (Fuchs, 2013a, p. 227-228).

Como resultado, o sujeito depressivo sofre de uma profunda despersonalização afetiva, apresentando uma aparência apática e uma postura prostrada. Seu rosto se revela de modo frequentemente inexpressivo; e seu olhar, cansado e vazio. Queixa-se da experiência de 'um sentimento de não sentir', percebendo-se inanimado e excluído de seu ambiente (Fuchs, 2005a). Há uma 
perda do impulso conativo-afetivo ou conação ${ }^{5}$, que vem a ser entendido por Fuchs (2012b) enquanto a "fonte inespecífica de energia presente em nossas atividades corporais" (p. 156 - tradução nossa) ou, como coloca em outros momentos, a própria sensação de estar vivo.

$\mathrm{Na}$ depressão, o vigor e a intensidade da experiência afetiva se extinguem, esvaecendo-se as buscas por prazer ou satisfação (Fuchs, 2005a; 2010a; Fuchs, $\&$ Schlimme 2009). A relação interafetiva entre homem e mundo é interrompida ou perturbada, contribuindo para uma experiência de imobilidade e solidão. A rigidez e a inibição do depressivo se contrastam com o movimento contínuo do mundo que o cerca, o que promove um sentimento de dissintonia temporal: enquanto a vida segue, o sujeito se vê fixo e paralisado (Fuchs, 2001a; 2005c; 2013c; 2018; Fuchs \& Duppen, 2017).

Diferentemente das concepções cognitivistas bastante difundidas no campo da saúde mental, a experiência depressiva é entendida por Fuchs (2002c; 2005a; 2005e; 2010a; 2013a; 2014b) como fundamentalmente corporal, e não estritamente mental. Ela se encontra no campo da relação homem-mundo, e não em pensamentos inscritos em um aparato cognitivo ou psíquico. Não há um modo de pensar do depressivo que anteceda e determine seu vivido, mas uma experiência pré-reflexiva do mundo que o constitui. Os ditos sintomas cognitivos não se configuram como o lócus do transtorno, mas enquanto resultante da experiência intercorporal antepredicativa.

Pensar o sujeito depressivo neste sentido implica em não considerar o transtorno como algo inato ou interno ao paciente, mas enquanto um fenômeno emergente nas relações intersubjetivas. Não há um homem interior, mas um homem que só é enquanto se relaciona ao mundo; que se apercebe e se conhece nesse mundo através do corpo (Merleau-Ponty, 1945/2011).

A depressão representa um modo de existir em que a nossa coexistência com os outros é drasticamente alterada, e esta alteração é vivida no corpo e através do corpo. Simultaneamente, Fuchs (2002a, 2002b, 2013a) não se apoia em um monismo reducionista que restringe a experiência ao fenômeno fisiológico e pensa o corpo de modo estritamente objetivista. Trata-se aqui de um corpo da experiência pré-reflexiva, que é expressão de nosso modo de estar no mundo, com os outros e consigo.

\footnotetext{
5 Advindo do latim conatus (impulso, tentativa, esforço), este termo usado por Fuchs foi primeiramente utilizado na filosofia estoica, sendo retomado por Hobbes e Spinoza posteriormente com o intuito de expressar o instinto do ser vivente e a autopreservação (conatus esse conservandi) (Fuchs, 2013c).
} 
A partir disso, Fuchs (2014) considera que o papel do tratamento da depressão é restabelecer a sincronia existente entre o paciente e seu mundo, aliando-se, a depender de cada caso, de intervenções psicoterapêuticas e psicofarmacológicas (Fuchs, 2002b). Koch e Fuchs (2011) também sinalizam para a importância da utilização de práticas psicoterapêuticas alternativas que explorem a movimentação corporal, não se restringindo apenas ao modelo de intervenção pela fala. Em um estudo desenvolvido por Koch, Morlinghaus e Fuchs (2007), os autores constatam os benefícios desse tipo de intervenção em pacientes depressivos ao verificar uma nítida melhoria no quadro dos sujeitos participantes de grupos de dança. Em contrapartida, Fuchs não apresenta muitas pesquisas referentes a terapias desse gênero em casos de depressão, deixando espaços a futuras pesquisas no campo.

\section{Considerações finais}

Identificamos na obra de Fuchs sua preocupação em construir uma proposta psicopatológica centrada na ideia de intersubjetividade, atrelando esta dimensão à intercorporeidade como ponte de constituição ambígua da relação homem/mundo, o que evidencia sua inspiração na proposta filosófica de MerleauPonty, ao radicalizar o entrelaçamento do homem ao mundo como elementos que não podem existir de forma separada.

Nesta perspectiva, o trabalho de Thomas Fuchs traz grandes contribuições para a psicopatologia fenomenológica contemporânea, sobretudo para a compreensão do fenômeno da depressão melancólica. Sempre evitando se centrar nos parâmetros diagnósticos ou nosológicos, Fuchs visa à compreensão de alterações presentes em uma das dimensões fundamentais da experiência, no caso, a corporeidade.

Esta dimensão é constituída de forma ambígua, apresentando uma face explícita e reflexiva, e outra intuitiva e tácita. É neste entrelaçamento que encontramos uma dinâmica intersubjetiva e irrestrita ao sujeito individual ao ser sempre compartilhada com os outros e com o mundo.

A corporeidade é um ponto chave nos escritos de Fuchs, sobretudo aqueles sobre a depressão melancólica, em que o paciente é compreendido como ser humano intercorpóreo, encarnado e como unidade indivisível e indissociável na experiência de ser no mundo. Destacamos a intersubjetividade do corpo, apresentada por Fuchs como intercorporeidade, como fio condutor para a compreensão do vivido depressivo e para o desenvolvimento de práticas terapêuticas que se utilizem do corpo como campo de intervenção. Ideia esta que 
se afasta radicalmente de uma compreensão dualista e dicotômica da experiência de adoecimento.

\section{Referências}

Bloc, L., Souza, C. \& Moreira, V. (2016). Phenomenology of depression: Contributions of Minkowski, Binswanger and Tatossian. Estudos de Psicologia, 33(1), 107-116. doi: 10.1590/1982-027520160001000011

Cutting, J., Mouratidou, M., Fuchs, T. \& Owen, G. (2016). Max Scheler's influence on Kurt Schneider. History of Psychiatry, 27(3), 336-344.

de Haan, S. \& Fuchs, T. (2010). The ghost in the machine: Disembodiment in schizophrenia - Two Cases Studies. Psychopathology, 43(5), 327-333. doi: $10.1159 \% 2 \mathrm{~F} 000319402$

Fuchs, T. (1994). Uprooting and late-life psychosis. European Archives of Psychiatry and Clinical Neuroscience, 222(3), 126-130.

Fuchs, T. (1999a). Life events in late paraphrenia and depression. Psychopathology, 32(2), 60-69.

Fuchs, T. (1999b). Patterns of relation and premorbid personality in late paraphrenia and depression. Psychopathology, 32(2), 70-80.

Fuchs, T. (2001a). Melancholia as a Desynchronazation: Towards a Psychopathology of Interpersonal Time. Psychopathology, 34 (4), 179-186. doi: $10.1159 / 000049304$

Fuchs, T. (2001b). The tacit dimension. Philosophy, Psychiatry \& Psychology, 8(4), 323-326. doi: 10.1353/ppp.2002.0018

Fuchs, T. (2002a). The challenge of neuroscience: Psychiatry and phenomenology today. Psychopathoology, 35(6), 319-326. doi: 10.1159/000068593

Fuchs, T. (2002b). Mind, meaning and the brain. Philosophy, Psychiatry and Psychology, 9(3), 261-264. doi: 10.1353/ppp.2003.0040

Fuchs, T. (2002c). The phenomenology of shame, guilt and the body in body dysphormic disorder and depression. Journal Phenomenological Psychology, 33(2), 223-243. doi: 10.1163/15691620260622903

Fuchs, T. (2005a). Corporealized and Disembodied Minds: A Phenomenological View of the Body in Melancholia and Schizophrenia. Philosophy, Psychiatry \& Psychology, 12(2), 95-107. 
Fuchs, T. (2005b). Delusional Mood and Delusional Perception - A phenomenological Analysis. Psychopathology, 38(3), 133-139. doi: $10.1159 / 000085843$

Fuchs, T. (2005c). Implicit and Explicit Temporality. Philosophy, Psychiatry \& Psychology, 12(3), 95-107. doi: 10.1353/ppp.2006.0004

Fuchs, T. (2005d). Overcoming Dualism. Philoshopy, Psychiatry \& Psychology, 12(2), 115-117.

Fuchs, T. (2005e). The phenomenology of body, space and time in depression. Comprendre, 15, 108-121.

Fuchs, T. (2006). Being a Psycho-Machine. On the phenomenology of the influencing-machine. Em T. Röske \& B. Brand-Claussen (Eds.). The Air Loom and Other Dangerous Influencing Machines (pp. 26-43). Heidelberg: Wunderhorn.

Fuchs, T. (2007a). Fragmented Selves: Temporality and Identity in Boderline personality disorder. Psychopathology, 40(6), 379-387. doi: 10.1159/000106468

Fuchs, T. (2007b) Psychotherapy of the Lived Space: A Phenomenological and Ecological. American Journal of Psychotherapy, 61(4), 423.

Fuchs, T. (2007c). The temporal structure of intetionality and its disturbance in schizophrenia. Psychopathology, 40(5), 229-235. doi: 10.1159/000101365

Fuchs, T. (2010a). Phenomenology and Psychopathology. Em S. Gallagher \& D. Schmicking (Eds.) Handbook of Phenomenology and Cognitve Science (pp. 547-573). Berlin: Springer. doi: 10.1007/978-90-481-2646-0_28

Fuchs, T. (2010b). The psychopathology of hyperreflexivity. Journal of Speculative Philosophy, 23(3), 239-255. doi: 10.1353/jsp.2010.0010

Fuchs, T. (2010c). Subjectivity and Intersubjectivity in Psychiatric Diagnosis. Psychopathology, 43(4), 268-274. doi: 10.1159/000315126

Fuchs, T. (2012a). Are mental Illnesses diseases of the brain? Em S. Choudhury, S. K. Nagel \& J. Slaby (Eds.) Critical Neuroscience: Linking Neuroscience and Society through Critical Practice (pp. 331-344). London: Blackwell.

Fuchs, T. (2012b). The Feeling of Being Alive. Organic foundations of selfawareness. Em J. Fingerhut \& S. Marienberg (Eds.). Feelings of Being Alive (pp. 149-165). Berlin/New York: De Gruyter. 
Fuchs, T. (2013a). Depression, Intercorporeality and interaffectivity. J. Consciousness Studies, 20(7-8), 219-238.

Fuchs, T. (2013b). Existential vulnerability: Toward a Psychopathology of Limit Situations. Psychopathology, 46(5), 301-308. doi: 10.1159/000351838

Fuchs, T. (2013c). Temporality and Psychopathology. Phenomenology and Cognitive Sciences, 12(1), 75-104. doi: 10.1007/s11097-010-9189-4

Fuchs, T. (2014a). Brain Mythologies. Jaspers' critique of reductionism from a current perspective. Em T. Fuchs, T. Breyer \& C. Mundt (Eds.). Karl Jaspers' Philosophy and Psychopathology (pp. 75-84). New York: Springer. doi: 10.1007/978-1-4614-8878-1

Fuchs, T. (2014b). Psychopathology of depression and mania: symptoms, phenomena and syndromes. Journal of Psychopathology, 20, 404-413.

Fuchs, T. (2015a). The Intersubjectivity of delusions. World Psychiatry, 14(2), 178-179.

Fuchs, T. (2015b). Pathologies of intersubjectivity in autism and schizophrenia. Journal of Conciousness Studies, 22(1-2), 191-214.

Fuchs, T. (2015c). From self-disorders to ego-disorders. Psychopathology, 48(5), 324-331. doi: 10.1159/000432404

Fuchs, T. (2018a). The Cyclical Time of the Body and its Relation to Linear Time. Journal of Conciousness Studies, 25(7-8), 47-65.

Fuchs, T. (2018b). Para uma psiquiatria fenomenológica: Ensaios e conferências sobre as bases antropológicas da doença psíquica, memória corporal e si mesmo ecológico (M. Casanova, Trad.). Rio de Janeiro: Via Verita.

Fuchs, T. \& De Jaegher, H. (2009). Enactive Intersubjectivity: Participatory sensemaking and mutual incorporation. Phenomenology and Cognitive Sciences, 8(4), 465-486. doi: 10.1007/s11097-009-9136-4

Fuchs, T. \& Duppen, Z. V. (2017). Time and Events: On the phenomenology of Temporal Experience in Schizophrenia (Ancillary Article to EAWE Domain 2). Psychopathology, 50(1), 68-74.

Fuchs, T. \& Koch, S. C. (2014). Embodied affectivity: on moving and being moved. Frontiers in Psychology, 5, 1-12. doi: 10.3389/fpsyg.2014.00508

Fuchs, T. \& Schlimme, J. (2009). Embodiment and psychopathology: a phenomenological perspective. Current Opinion in Psychiatry, 22(6), 570575. doi: 10.1097/YCO.0b013e3283318e5c 
Gaete, M. I. \& Fuchs, T. (2016). From body image to emotional bodily experience in eating disorders. Journal of Phenomenological Psychology, 47(1), 17-40. doi: $10.1163 / 15691624-12341303$

Hirjak, D., Breyer, T., Thomann, P. A. \& Fuchs, T. (2013). Disturbance of Intentionality: A Phenomenological Study of Body-Affecting First-Rank Symptoms in Schizophrenia. PloS one, 8(9), 1-10. doi: 10.1371/journal.pone.0073662

Husserl, E. (2014). A crise das ciências europeias e a fenomenologia transcendental: uma introdução à filosofia fenomenológica (D. Ferrer, Trad.). Rio de Janeiro: Forense Universitária. (Original publicado em 1954).

Koch, S. C., \& Fuchs, T. (2011). Embodied arts therapies. The Arts in Psychotherapy, 38(4), 276-280. 10.1016/j.aip.2011.08.007

Koch, S. C., Morlinghaus, K., \& Fuchs, T. (2007). The joy dance: Specific effects of a single dance intervention on psychiatric patients with depression. The Arts in Psychotherapy, 23(4), 340-349. doi: 10.1016/j.aip.2007.07.001

Merleau-Ponty, M. (2006). A Estrutura do Comportamento (M. V. Aguiar, Trad.). São Paulo: WMF Martins Fontes. (Original publicado em 1942).

Merleau-Ponty, M. (2011). Fenomenologia da Percepção (C. A. Moura, Trad.). São Paulo: WMF Martins Fontes. (Original publicado em 1945).

Merleau-Ponty, M. (2014). O Visível e o Invisível (J. A. Gianotti \& A. d'Oliveira, Trad.). São Paulo: Perspectiva. (Original publicado em 1964).

Minkowski, E. (1997). La schizophrénie: psychopathologie des schizoïdes et des schizophrènes. Paris : Payot \& Rivages. (Original publicado em 1927).

Reck, C. e outros (2004). Interactive regulation of affect in postpartum depressed mother and their infants: An overview. Psychopathology, 37(6), 272-280.

Reck, C. e outros (2008). Prevalence, onset and comorbidty of postpartum anxiety and depressive disorders. Acta Psychiatrica Scandinavica, 118(6), 459-468.

Reck, C. e outros (2011). Interactive coordination of currently depressed inpatient mother and their infants during the postpartum period. Infant Mental Health Journal, 32(5), 542-562.

Tatossian, A. (2006). A Fenomenologia das Psicoses (C. Freire, Trad.). São Paulo: Escuta. (Original publicado em 1979). 
WHO, (2018). Depression. Recuperado em 30 de setembro, 2018, de http://www.who.int/news-room/fact-sheets/detail/depression.

\section{Nota sobre os autores}

Victor Monteiro é graduando em Psicologia da Universidade de Fortaleza. Possui formação em Psicoterapia Humanista Fenomenológica. Membro do Laboratório de Psicopatologia e Clínica Humanista Fenomenológica - APHETO. Email: vmonteiroleite@gmail.com

Camila Souza é psicóloga. Mestre e Doutora em Psicologia pela Universidade de Fortaleza. Atualmente, é pesquisadora do Laboratório de Psicopatologia e Clínica Humanista Fenomenológica - APHETO. Docente do curso de Psicologia do Centro Universitário Farias Brito, onde coordena o Centro de Atendimento Psicológico. E-mail: camila psouza@hotmail.com

Virgínia Moreira é Mestre em Educação pela Universidade Federal do Ceará. Doutora em Psicologia Clínica pela Pontifícia Universidade Católica de São Paulo. Psicoterapeuta com formação na Enfoque Centrada na Pessoa, pelo Center of Studies of the Person (La Jolla, EUA). Membro do GT Psicologia \& Fenomenologia da ANPEPP. Atualmente, é professora-titular do Programa de Pós-Graduação em Psicologia da Universidade de Fortaleza, onde coordena o Laboratório de Psicopatologia e Clínica Humanista Fenomenológica - APHETO. E-mail: virginiamoreira@unifor.br

Data de recebimento: 08 de agosto de 2019

Data de aceite: 11 de novembro de 2020 